\title{
On solving fuzzy delay differential equation using bezier curves
}

\author{
Ali F. Jameel ${ }^{1}$, Sardar G. Amen², Azizan Saaban³, Noraziah H. Man ${ }^{4}$ \\ ${ }^{1,2,3,4}$ School of Quantitative Sciences, College of Art and Sciences, Universiti Utara Malaysia (UUM), Malaysia \\ ${ }^{2}$ Department of Financial and Banking, Collage of Business Administration and Financial Science, \\ Al-Kitab University, Iraq
}

\begin{tabular}{|c|c|}
\hline Article Info & ABSTRACT \\
\hline Article history: & In this article, we plan to use Bezier curves method to solve linear fuzzy \\
\hline Received Apr 14, 2020 & $\begin{array}{l}\text { delay differential equations. A Bezier curves method is presented and } \\
\text { modified to solve fuzzy delay problems taking the advantages of the fuzzy }\end{array}$ \\
\hline Revised May 27, 2020 & set theory properties. The approximate solution with different degrees is \\
\hline Accepted Jun 12, 2020 & $\begin{array}{l}\text { compared to the exact solution to confirm that the linear fuzzy delay } \\
\text { differential equations process is accurate and efficient. Numerical example is }\end{array}$ \\
\hline Keywords: & $\begin{array}{l}\text { explained and analyzed involved first order linear fuzzy delay differential } \\
\text { equations to demonstrate these proper features of this proposed problem. }\end{array}$ \\
\hline
\end{tabular}

Bezier control points

Delay differential equations

Fuzzy differential equations

Fuzzy numbers

Fuzzy set theory

Residual function

Copyright (C) 2020 Institute of Advanced Engineering and Science. All rights reserved.

\section{Corresponding Author:}

Ali F. Jameel,

School of Quantitative Sciences, College of Art and Sciences,

Universiti Utara Malaysia (UUM),

Sintok, 06010 Kedah, Malaysia.

Email: alifareed@uum.edu.my

\section{INTRODUCTION}

Fuzzy set theory is a powerful instrument for modeling uncertainty in a wide range of real issues and for processing vague or subjective information in mathematical patterns. DDEs are a type of differential equation in which the derivative of the unknown function at a certain time is given in terms of the values of the function at a previous time. Often called DDEs time-delay systems with or with dead impact-time, inherited process equations with deviating argument $[1,2]$.

The fundamental theory of steady works and key theory variables such as unique solutions are found in [1-3]. Next, a large number of the Delay Differential Equation have been extensively investigated in the novel, and monographs were published, including considerable on in [4], and so forth. The research advantage of the differential delays is because many systems have been the prototype of better differential delays in engineering, economics, science, etc. The difference equations of delays are delayed. Nevertheless, they are not realistic to regulate problems. Most of these equations obviously cannot be precisely solved. Efficient numerical methods must therefore be designed to approach their solutions. Ishiwata et al. used the rational approximation method and the collocation method [5-7] to compute numerical solutions of DDEs with proportional delays. Hu et al. [8] applied linear multi-step methods to compute numerical solutions for neutral DDEs. Other method obtained approximate solutions for variety of DDEs such as Runge Kutta methods, block methods and one- leg $\theta$-methods in [8-12]. Moreover, the DDEs solved approximately via some approximation methods in many fields of mathematics using approximation methods: for example, the homotopy analysis method [13, 14], Adomian decomposition method [15] and homotopy perturbation 
method [16]. Fuzzy DDE problem will model when the crisp model is not complete and its premasters or conditions under fuzzy properties. FDDEs were solved by multiple researchers in recent years with an approximate solution in $[17,18]$. We will present in this article new plans for the approximate solution of FDDEs by means of the curves of Bezier method in fuzzy domain and analyzed the fuzzy solutions in different degree of approximations.

The outline of this paper will be as follows: FDDEs will be introduced in section 2. In section 3, Problem of Fuzzy Delay System will be declared. Introduced proportional delay with FDDEs in section 4. In sections 5 and 6 respectively degree elevation and Bezier curves and will be declared. Using Bezier control points for Solving FDDE aforementioned method and suggested will be implemented on it in section 7. In section 8 solved numerical problems, appeared the accuracy and adequacy of the method. Lastly, the conclusion briefly will be given in section 9 .

\section{DESCRIPTION OF DELAY FUZZY DIFFERENTIAL EQUATIONS}

Many DDEs are increasing, fundamentally optimistic in the models of epidemiology and population dynamics. It is therefore worth noting that positive initial data lead to positive solutions [15]. Consider the following FDDE:

$$
D \tilde{v}(x)=\tilde{y}(x, \tilde{v}(x), \tilde{v}(x-k))
$$

where for all fuzzy level sets $r \in[0,1]$ we have the following defuzzifications:

- The fuzzy functions $\tilde{v}(x)[19]$ is denoted as $\tilde{v}(x ; r)=[\underline{v}(x ; r), \bar{v}(x ; r)]$,

- The fuzzy delay functions $\tilde{v}(x-k)$ is denoted as $\tilde{v}(x-\alpha ; r)=[\underline{v}(x-\alpha ; r), \bar{v}(x-\alpha ; r)]$

- $\quad$ The fuzzy first order H-derivative, see [19]

$$
D \tilde{v}(x ; r)=[D \underline{v}(x ; r), D \bar{v}(x ; r)] \text {, }
$$

Next, assume that the fuzzy function in (1) can be written as:

$$
\begin{aligned}
& \tilde{y}(x, \tilde{v}(x), \tilde{v}(x-\alpha))=\tilde{y}(x, \tilde{V}(x)) \text { such that } \\
& \tilde{y}(t, \tilde{V}(x))=[\underline{y}(t, \underline{\tilde{V}}(x)), \underline{y}(t, \overline{\tilde{V}}(x))]
\end{aligned}
$$

By using Zadeh extension principles [20], we have the following membership function

$$
\begin{aligned}
& F(x, \tilde{V}(x ; r))=\min \left\{D \tilde{v}(x ; r): \mu \mid \mu \in[\tilde{V}(x)]_{r}\right\}, \\
& G(x, \tilde{V}(x ; r))=\max \left\{D \tilde{v}(x ; r): \mu \mid \mu \in[\tilde{V}(x)]_{r}\right\},
\end{aligned}
$$

where

$$
\left\{\begin{array}{l}
\underline{y}(x, \tilde{V}(x ; r))=F(t, \underline{V}(x ; r), \bar{V}(x ; r))=F(x, \tilde{V}(x ; r)) \\
\bar{y}(x, \tilde{V}(x ; r))=G(t, \underline{V}(x ; r), \bar{V}(t ; r))=G(x, \tilde{V}(x ; r))
\end{array}\right.
$$

with a single delay $k>0$. For each $r \in[0,1]$, suppose that $[\tilde{y}(x, \tilde{V})]_{r}$ and $\left[\tilde{y}_{v}(x, \tilde{V})\right]_{r}$, are continuous on $\mathbb{R}^{3}$. Let $\varphi:[z-k, z] \rightarrow \mathbb{R}$ be continuous where $z \in \mathbb{R}$ be given. Require the solution $v(x)$ of (1) satisfying

$$
\tilde{v}(x ; r)=\tilde{\varphi}(x ; r), z-k \leq x \leq z
$$

and satisfying (1) on $z \leq x \leq z+\alpha$ for some $\alpha>0$. Note: should be explain $D \tilde{v}(x ; r)$ as the right-hand derivative at $z$. Now demonstrate a material system design problem that shows phenomenon of time delay. The question picked in this section is exactly the right one in the test (1). 


\section{PROBLEM OF TIME FUZZY DELAY SYSTEM}

The existence of lags in economic systems is completely normal since a decision for the results of article should be given a fixed period after. In one sample [21] of total economy and suppose $\widetilde{U}(x)$ be the proceeds which can divide into autonomous expenditure, consumption $\tilde{A}(x)$, and investment $\widetilde{B}(x)$. From section 2, we have:

$$
\begin{aligned}
\widetilde{U}(x ; r) & =\tilde{A}(x ; r)+\tilde{B}(x ; r)+\tilde{C}(x ; r) \\
\tilde{A}(x ; r) & =\tilde{b} \widetilde{U}(x ; r)
\end{aligned}
$$

where $\tilde{b}$ is a consumption fuzzy coefficient following the properties of triangular fuzzy number [17]. From (4),

$$
\widetilde{U}(x ; r)=\frac{\tilde{B}(x ; r)+\tilde{C}(x ; r)}{1-\tilde{b}}\left\{\begin{array}{c}
\frac{\underline{B}(x ; r)+\underline{C}(x ; r)}{1-\underline{b}(r)} \\
\frac{\bar{B}(x ; r)+\bar{C}(x ; r)}{1-\bar{b}(r)}
\end{array}\right.
$$

Assuming that, following a decision to run $\widetilde{D}(x)$ there is limited time between the production and ordering of capital instruments. In expression of the paper of capital savings $\tilde{J}(x)$, we have

$$
\begin{aligned}
& \tilde{J}^{\prime}(x ; r)=\widetilde{D}(x-k ; r) \\
& \tilde{B}(x ; r)=\frac{1}{k} \int_{x-k}^{x} \widetilde{D}(T ; r) d T
\end{aligned}
$$

For each fuzzy level set $r \in[0,1]$ in crisp domain the economic rationale suggests that $\widetilde{D}(x ; r)$ is given by rate of saving proportionate to $\widetilde{U}(x ; r)$ and by the capital paper $\tilde{J}(x ; r)$ such that

$$
\widetilde{D}(x ; r)=\gamma(1-\tilde{b}) \widetilde{U}(x ; r)-\delta \tilde{J}(x ; r)+\rho
$$

where $\gamma>0, \delta>0$ and $\rho$ is direction factor. Combining (6) and (7) to obtain the following

$$
\tilde{B}(x ; r)=\frac{1}{k}[\tilde{J}(x+k ; r)-\tilde{J}(x ; r)]
$$

From (6) and (9), we get

$$
\widetilde{U}(x ; r)=\frac{1}{k(1-\tilde{b})}[\tilde{J}(x+k ; r)-\tilde{J}(x ; r)]+\frac{\tilde{C}(x ; r)}{1-\tilde{b}}
$$

By combining (8)-(10), we can yield

$$
\dot{\tilde{J}}(x ; r)=\frac{\gamma}{k} \tilde{J}^{\prime}(x ; r)-\left(\delta+\frac{\gamma}{k}\right) \tilde{J}(x+k ; r)+[\gamma \tilde{C}(x ; r)+\rho]
$$

Express acceptance rate new appointment information. It is a delayed-type template operational FDDE.

\section{FDDES WITH PROPORTIONAL DELAY}

In this research, the Bezier control point method can finish off approximate analytical solutions with a high level of reliability. Consider the following neutral functional FDEE with proportional delays [21, 22],

$$
\left(\tilde{v}(x ; r)+\tilde{a}(x ; r) \tilde{v}\left(p_{k} x ; r\right)\right)^{n}=\tilde{\beta} \tilde{v}(x ; r)+\sum_{k=0}^{n-1} \tilde{b}_{k}(x ; r) \tilde{v}^{(k)}\left(p_{k} x ; r\right)+\tilde{y}(x ; r)
$$

with the fuzzy initial conditions

$$
\begin{aligned}
& \sum_{k=0}^{n-1} \tilde{c}_{i k} \tilde{v}^{(k)}(0 ; r)=\tilde{\delta}_{i}(r), \\
& i=0,1, \ldots, n-1 .
\end{aligned}
$$


Here, $\tilde{a}(x ; r)$ and $\tilde{b}_{k}(x ; r)(k=0,1, \ldots, n-1) \mathrm{k}$ are given analytical fuzzy functions, and $\tilde{\beta}, \tilde{p}_{k}, \tilde{c}_{i k}$, and $\check{\delta}_{i}$ denote given fuzzy constants with $0<p_{k}<1,(k=0,1, \ldots, n)$. The presence and singularity of the multi pantograph equation analytical solution is demonstrated [21], the solution Dirichlet sequence is constructed and the asymptotic stability of the analytical solution is sufficiently defined.. It is proved that the $\theta$-methods with a variable step size are asymptotically stable if $\frac{1}{2}<\theta \leq 1$. There are several examples that show the properties of the $\theta$-methods. In order to apply the Bezier control point method, we rewrite (12) as

$$
(\tilde{v}(x ; r))^{n}=\beta \tilde{v}(x ; r)-\left(a(x) v\left(p_{k} x\right)\right)^{n}+\sum_{k=0}^{n-1} b_{k}(x) v^{(k)}\left(p_{k} x\right)+y(x), x \geq 0
$$

A particular class of crisp DDE represents neutral functional DEEs with proportional delays. The mathematical modeling of real-world phenomena takes such functioning DEEs on a significant role [12].

\section{BEZIER CURVES IN FUZZY DOMAIN}

From the definition of Bezier curve polynomial of $m$ degree [23] and according to sections 2-4, we have the following fuzzy analysis

$$
\begin{aligned}
& \tilde{B}(x ; r)=\sum_{j=0}^{m} \tilde{P}_{j} \widetilde{B}_{j}^{m}\left(\frac{x-b_{1}}{b_{2}-b_{1}} ; r\right), x \in\left[b_{1}, b_{2}\right] . \\
& \tilde{B}_{j}^{m}\left(\frac{x-b_{1}}{b_{2}-b_{1}} ; r\right)=\frac{m !}{j !(m-j) !}\left(\frac{x-b_{1}}{b_{2}-b_{1}} ; r\right)^{j}\left(\frac{b_{2}-x}{b_{2}-b_{1}} ; r\right)^{m-j}
\end{aligned}
$$

$P_{j}$ is control points of Bezier coefficient and $\tilde{B}_{j}^{m}$ are the polynomial of Bernstein on interval [a, $\mathrm{a}_{2}$ ] per each fuzzy level set $r \in[0,1]$, see Figure 1. In particular

$$
\begin{aligned}
& \tilde{B}(x ; r)=\sum_{j=0}^{m} P_{j} \tilde{B}_{j}^{m}(x ; r), x \in[0,1] . \\
& \tilde{B}_{j}^{m}(x ; r)=\frac{m !}{j !(m-j) !}(x ; r)^{j}(1-x ; r)^{m-j}
\end{aligned}
$$

where $\tilde{B}(x ; r)$ is a fuzzy parametric Bezier curve when it's polynomial of vector valued. Figure 1 shows the comprise line segments with control polygon of a Bezier curve $C_{j}-C_{j+1}, \mathrm{j}=0,1, \ldots, \mathrm{m}-1$. If $\tilde{B}(x ; r)$ polynomial of a scalar valued, the function is call $\tilde{y}=\tilde{B}(x ; r)$ then from $[23,24]$ an explicit Bezier curve denoted by $(x, \tilde{B}(x ; r))$.

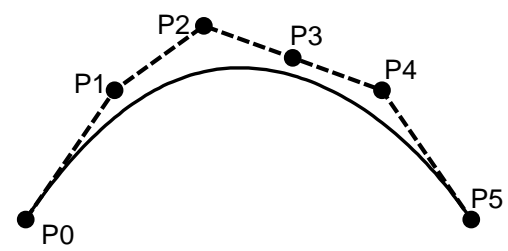

Figure 1. Degree 5 bezier curve with control polygon

\section{SOLUTION OF FDDE USING BEZIER CONTROL POINTS}

Consider the following boundary value problem

$$
\begin{aligned}
& L\left(\tilde{v}(x ; r), \tilde{v}\left(p_{0} x ; r\right), \ldots, \tilde{v}\left(p_{n} x ; r\right)\right) \tilde{v}^{(n)}(x ; r)-\beta \tilde{v}(x ; r)+\left(a(x) \tilde{v}\left(p_{n} x ; r\right)^{(n)}-\right. \\
& \sum_{k=0}^{n-1} \tilde{b}_{k}(x ; r) \tilde{v}^{(k)}\left(p_{k} x ; r\right)+\tilde{y}(x ; r),, x \geq 0 \\
& \frac{d^{j} \tilde{v}(0 ; r)}{d x^{j}}=a_{j}, \frac{d^{j} \tilde{v}(1 ; r)}{d x^{j}}=\beta_{j}, j=0,1, \ldots, n-1,
\end{aligned}
$$


where $L$ is differential operator with proportional delay, is $\tilde{y}(x ; r)$ also a polynomial in $x, 0<p_{k}<1$ and $(\mathrm{k}=0,1, \cdots, \mathrm{m})[24,25]$. We propose to represent the approximate solution of eq. (18) $\tilde{v}(x ; r)$ in fuzzy Bezier form. The preference between Bezier and B-Spline is that the Bezier form is easier to carry out multiplication, contrast and degree elevation operations symbolically than B-Spline. We choose the sum of squares of the Bezier control points of the residual to be the measure quantity. Minimizing this quantity gives the approximate solution. Therefore, the obvious spotlight is in the following, if the minimizing of the quantity is zero, so the residual function is zero, which implies that the solution is the exact solution. We call this approach the control point based method. By following [25] detailed steps of the method are as follows:

Step 1 . Choose a degree $\mathrm{n}$ and symbolically express the solution $\tilde{v}(x ; r)$ in the degree $\mathrm{m}(m \geq n)$ Bezier form

$$
\tilde{v}=\tilde{v}(x ; r)=\sum_{j=0}^{m} \tilde{\alpha}_{j}(x ; r) \tilde{B}_{j}^{m}(x ; r)
$$

where the control points are $\alpha_{0}, \alpha_{1}, \ldots, \alpha_{m}$ to be de-termined.

Step 2. Substituting the approximate solution $v=v(x)$ into the (19), we obtain the residual function

$$
\tilde{R}(x ; r)=L\left(\tilde{v}(x ; r), \widetilde{v}\left(p_{0} x\right), \widetilde{v}\left(p_{1} x ; r\right), \ldots, \widetilde{v}\left(p_{n} x ; r\right)\right)-\tilde{y}(x ; r) .
$$

This is a polynomial in $x$ with degree $\leq \mathrm{h}$, where

$$
\begin{aligned}
& h=\max \left\{m-n+\operatorname{deg}\left(\tilde{\alpha}(x ; r), m+\operatorname{deg}\left(\tilde{b}_{0}(x ; r)\right), m-1+\operatorname{deg}\left(\tilde{b}_{1}(x ; r)\right), \ldots, m-n+1+\right.\right. \\
& \left.\operatorname{deg}\left(\tilde{b}_{n-1}(x ; r)\right), \operatorname{deg}(\tilde{y}(x ; r))\right\} .
\end{aligned}
$$

So the residual function $\tilde{R}(x ; r)$ can be expressed in fuzzy Bezier form as well,

$$
\tilde{R}=\tilde{R}(x ; r)=\sum_{j=0}^{h} \tilde{b}_{j} \tilde{B}_{j}^{h}(x)
$$

where for each fuzzy level set $r \in[0,1]$ the control points $\tilde{b}_{0}, \tilde{b}_{1}, \ldots, \tilde{b}_{h}$ are linear functions in the unknowns $\tilde{\alpha}_{j}$. These functions are derived using the operations of multiplication, degree elevation and differentiation for Bezier form.

Step 3. Construct the objective function

$$
\tilde{F}(x ; r)=\sum_{j=0}^{h} \tilde{b}_{j}^{2}(x ; r) .
$$

Then $\tilde{F}(x ; r)$ is also a fuzzy function of $a_{0}, a_{1}, \ldots, a_{m}$.

Step 4. Solve the constrained optimization problem:

$$
\begin{aligned}
& \min \tilde{F}(x ; r)=\sum_{j=0}^{h} \tilde{b}_{j}^{2}\left(a_{0}, a_{1}, \ldots, a_{m} ; r\right), \\
& \frac{d^{j} \tilde{v}(0 ; r)}{d x^{j}}=a_{j}, \frac{d^{j} \tilde{v}(1 ; r)}{d x^{j}}=\tilde{\beta}_{j}, j=0,1, \ldots, n-1,
\end{aligned}
$$

by some optimization techniques, such as Lagrange multipliers method, we can be used to solve (21).

Step 5. Substituting the minimum solution back into (19) arrives at the approximate solution to the differential equation.

\section{NUMERICAL EXAMPLE}

In this part, we used the mentioned control-point-based method on Bezier control points to solve DDE's and system of DDE's. As a practical example, we consider Evens and Raslan [6] the following pantograph delay equation in fuzzy form:

$$
\begin{aligned}
& \tilde{u}^{\prime}(x)=\frac{1}{2} \exp \left(\frac{x}{2}\right) \tilde{u}\left(\frac{x}{2}\right)+\frac{1}{2} \tilde{u}(x), 0 \leq x \leq 1, \\
& \tilde{u}(0)=[r .2-r] .
\end{aligned}
$$


The exact solution is given by $\underline{u}(x ; r)=r e^{x}, \bar{u}(x ; r)=(2-r) e^{x}$.

According to [5] (21) can be written in defuzzfication form

$$
\left\{\begin{array}{c}
\underline{u}^{\prime}(x ; r)=\frac{1}{2} \exp \left(\frac{x}{2} ; r\right) \underline{u}\left(\frac{x}{2} ; r\right)+\frac{1}{2} \underline{u}(x ; r), \\
\underline{u}(0 ; r)=r \\
\bar{u}^{\prime}(x ; r)=\frac{1}{2} \exp \left(\frac{x}{2} ; r\right) \bar{u}\left(\frac{x}{2} ; r\right)+\frac{1}{2} \bar{u}(x ; r), \\
\bar{u}(0 ; r)=2-r
\end{array}\right.
$$

For numerical implementation, we consider the approximate solution using Bezier curves of degree $3(\mathrm{~m}=3)$ and $8(\mathrm{~m}=8)$ respectively as given in $(15)$. In order to obtain the residual function, we also approximate $\mathrm{e}^{x}$ in Taylor polynomial of order 6 . The detail results are as follows.

\subsection{Degree-3 Bezier curve}

$$
\left\{\begin{array}{l}
\underline{u}(x ; r)=\sum_{i=1}^{3} \underline{a}_{i} B_{i}^{3}(x) \\
\bar{u}(x ; r)=\sum_{i=1}^{3} \bar{a}_{i} B_{i}^{3}(x)
\end{array}\right.
$$

where $0 \leq x \leq 1$ and $\underline{a}_{i}$ and $\bar{a}_{i}, i=0, \ldots, 3$ are the Bezier control points need to be determined. Substitute into (23) and the residual functions can be obtained, i.e.

$$
\left\{\begin{aligned}
\underline{R}(x ; r) & =\frac{d}{d x}\left(\sum_{i=1}^{3} \underline{a}_{i} B_{i}^{3}(x)\right)-\frac{1}{2} \exp \left(\frac{x}{2} ; r\right)\left(\sum_{i=1}^{3} \underline{a}_{i} B_{i}^{3}\left(\frac{x}{2}\right)\right) \\
& \left.-\sum_{i=1}^{3} \underline{a}_{i} B_{i}^{3}(x)\right) \\
\bar{R}(x ; r) & =\frac{d}{d x}\left(\sum_{i=1}^{3} \bar{a}_{i} B_{i}^{3}(x)\right)-\frac{1}{2} \exp \left(\frac{x}{2} ; r\right)\left(\sum_{i=1}^{3} \bar{a}_{i} B_{i}^{3}\left(\frac{x}{2}\right)\right) \\
& \left.-\sum_{i=1}^{3} \bar{a}_{i} B_{i}^{3}(x)\right)
\end{aligned}\right.
$$

The right-hand side of (25) is a polynomial of degree 8 and therefore the residual function can be represented in the form of (20) with $h=8$ as follows.

$$
\left\{\begin{array}{l}
\underline{R}(x ; r)=\sum_{i=0}^{8} \underline{b}_{i} B_{i}^{8}(x) \\
\bar{R}(x ; r)=\sum_{i=0}^{8} \bar{b}_{i} B_{i}^{8}(x)
\end{array}\right.
$$

To obtain the Bezier control points in (24), we follow the step 3 to step 5 as stated in section 6 . The approximate solutions are available in Tables 1 and 2 and the comparsion of degree 3 bezier curve solution with exact solution of equation (22) is illustrated in Figure 2 such that:

$$
\begin{aligned}
& \underline{u}(x ; r)=r\left[(1-x)^{3}+4.0126515(1-x)^{2} x+5.4528665(1-x) x^{2}\right. \\
& \left.\left.+2.71821024 x^{3}\right)\right] \\
& \bar{u}(x ; r)=(2-r)(1-x)^{3}+(8.025303-4.0126516 r)(1-x)^{2} x+ \\
& (1.090573311-5.4528665 r)(1-x) x^{2}+ \\
& \left.\left.(4.43642047-2.71821024 r) x^{3}\right)\right]
\end{aligned}
$$


Table 1. Appoximate and exact values for lower solution,

\begin{tabular}{|c|c|c|c|}
\hline $\mathrm{r}$ & approx & exact & abs. error \\
\hline 0 & 0 & 0 & 0 \\
\hline 0.2 & 0.5436420472 & 0.5436563657 & $1.4318499128 \times 10^{-5}$ \\
\hline 0.4 & 1.0872840944 & 1.0873127314 & $2.8636998257 \times 10^{-5}$ \\
\hline 0.6 & 1.6309261416 & 1.6309690971 & $4.2955497386 \times 10^{-5}$ \\
\hline 0.8 & 2.1745681888 & 2.1746254628 & $5.7273996514 \times 10^{-5}$ \\
\hline 1.0 & 2.718210236 & 2.7182818285 & $7.1592495642 \times 10^{-5}$ \\
\hline
\end{tabular}

Table 2. Approximate and exact values for upper solution, $\bar{u}(x ; r)$ (degree 3 bezier curve)

\begin{tabular}{cccc}
\hline $\mathrm{r}$ & approx & exact & abs. error \\
\hline 0 & 5.436420472 & 5.4365636569 & $1.4318499128 \times 10^{-4}$ \\
0.2 & 4.892778425 & 4.8929072912 & $1.2886649216 \times 10^{-4}$ \\
0.4 & 4.349136378 & 4.3492509255 & $1.1454799303 \times 10^{-4}$ \\
0.6 & 3.805494330 & 3.8055945598 & $1.002294939 \times 10^{-4}$ \\
0.8 & 3.261852283 & 3.2619381942 & $8.591099477 \times 10^{-5}$ \\
1.0 & 2.71821024 & 2.7182818285 & $7.159249564 \times 10^{-5}$ \\
\hline
\end{tabular}

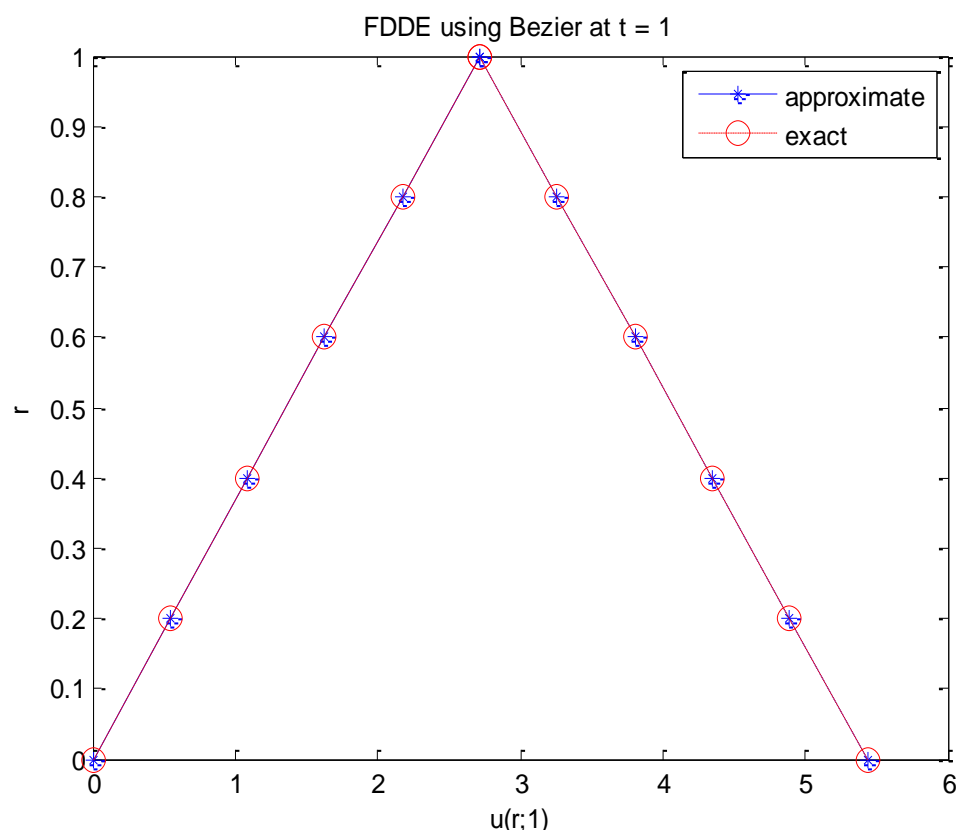

Figure 2. Approximate and exact solution of $u(t)$ at $\mathrm{t}=1$ (degree 3 bezier curves)

\subsection{Degree-8 Bezier curve}

Let,

$$
\left\{\begin{array}{l}
\underline{u}(x ; r)=\sum_{i=0}^{8} \underline{a}_{i} B_{i}^{8}(x) \\
\bar{u}(x ; r)=\sum_{i=0}^{8} \bar{a}_{i} B_{i}^{3}(x)
\end{array}\right.
$$

where $0 \leq x \leq 1$ and $\underline{a}_{i}$ and $\bar{a}_{i}, i=0, \ldots, 8$ are the Bezier control points need to be determined. Substitute into (23) and the residual functions can be obtained, i.e. 


$$
\left\{\begin{aligned}
\underline{R}(x ; r)= & \frac{d}{d x}\left(\sum_{i=0}^{8} \underline{a}_{i} B_{i}^{3}(x)\right)-\frac{1}{2} \exp \left(\frac{x}{2} ; r\right)\left(\sum_{i=0}^{8} \underline{a}_{i} B_{i}^{8}\left(\frac{x}{2}\right)\right) \\
& \left.-\sum_{i=0}^{8} \underline{a}_{i} B_{i}^{8}(x)\right) \\
\bar{R}(x ; r)= & \frac{d}{d x}\left(\sum_{i=0}^{8} \bar{a}_{i} B_{i}^{8}(x)\right)-\frac{1}{2} \exp \left(\frac{x}{2} ; r\right)\left(\sum_{i=0}^{8} \bar{a}_{i} B_{i}^{8}\left(\frac{x}{2}\right)\right) \\
& \left.-\sum_{i=0}^{8} \bar{a}_{i} B_{i}^{8}(x)\right)
\end{aligned}\right.
$$

The right-hand side of (25) is a polynomial of degree 13 and therefore the residual function can be represented in the form of (20) with $h=13$ as follows.

$$
\left\{\begin{array}{l}
\underline{R}(x ; r)=\sum_{i=0}^{13} \underline{b}_{i} B_{i}^{13}(x) \\
\bar{R}(x ; r)=\sum_{i=0}^{13} \bar{b}_{i} B_{i}^{13}(x)
\end{array}\right.
$$

To obtain the Bezier control points in (27), we also use the step 3 to step 5 as stated in section 6.

\begin{tabular}{|c|c|c|c|}
\hline $\mathrm{r}$ & approx & exact & abs. error \\
\hline 0 & 0 & 0 & 0 \\
\hline 0.2 & 0.543655820 & 0.5436563657 & $5.45269859 \times 10^{-7}$ \\
\hline 0.4 & 1.087311648 & 1.0873127314 & $1.090539719 \times 10^{-6}$ \\
\hline 0.6 & 1.630967461 & 1.6309690971 & $1.635809578 \times 10^{-6}$ \\
\hline 0.8 & 2.174623282 & 2.1746254628 & $2.181079437 \times 10^{-6}$ \\
\hline 1.0 & 2.718279102 & 2.7182818285 & $2.726349297 \times 10^{-6}$ \\
\hline
\end{tabular}
The approximate solutions are in Tables 3 and 4 and the comparsion of degree 8 bezier curve solution with exact solution of equation (22) is illustrated n Figure 3 such that:

$$
\begin{aligned}
& \underline{u}(x ; r)=r\left[(1-t)^{8}+9 t(1-t)^{7}+\right. \\
& 35.5000002 t^{2}(1-t)^{6}+80.16666616 t^{3}(1-t)^{5}+ \\
& 113.3750016 t^{4}(1-t)^{4}+102.84166368 t^{5}(1-t)^{3}+ \\
& 58.44305288 t^{6}(1-t)^{2}+ \\
& \left.19.0279716 t^{7}(1-t)+2.7182791 t^{8}\right] \\
& \bar{u}(x ; r)=(2-r)(1-t)^{8}+(1.8-9 r) t(1-t)^{7}+ \\
& (71.00000012-3.5 .5000002 r) t^{2}(1-t)^{6}+ \\
& (160.33333232-80.16666616 r) t^{3}(1-t)^{5}+ \\
& (226.7500039-113.3750016 r) t^{4}(1-t)^{4}+ \\
& (205.68332736-102.84166368 r) t^{5}(1-t)^{3}+ \\
& (116.8861176-58.4430588 r) t^{6}(1-t)^{2}+ \\
& (38.0559432-19.0279716 r) t^{7}(1-t)+ \\
& (5.4365582-2.7182791 r) t^{8}
\end{aligned}
$$

Table 3. Approximate and exact values for lower solution, 
Table 4. Approximate and exact values for upper solution, $\bar{u}(x ; r)$ (degree 8 bezier curve)

\begin{tabular}{cccc}
\hline $\mathrm{r}$ & approx & exact & abs. error \\
\hline 0 & 5.4365582042 & 5.4365636569 & $5.452698593 \times 10-6$ \\
0.2 & 4.8929023838 & 4.8929072912 & $4.907428734 \times 10-6$ \\
0.4 & 4.3492465634 & 4.3492509255 & $4.362158874 \times 10-6$ \\
0.6 & 3.8055907430 & 3.8055945598 & $3.816889015 \times 10-6$ \\
0.8 & 3.2619349225 & 3.2619381941 & $3.271619156 \times 10-6$ \\
1.0 & 2.7182791021 & 2.7182818284 & $2.726349297 \times 10-6$ \\
\hline
\end{tabular}

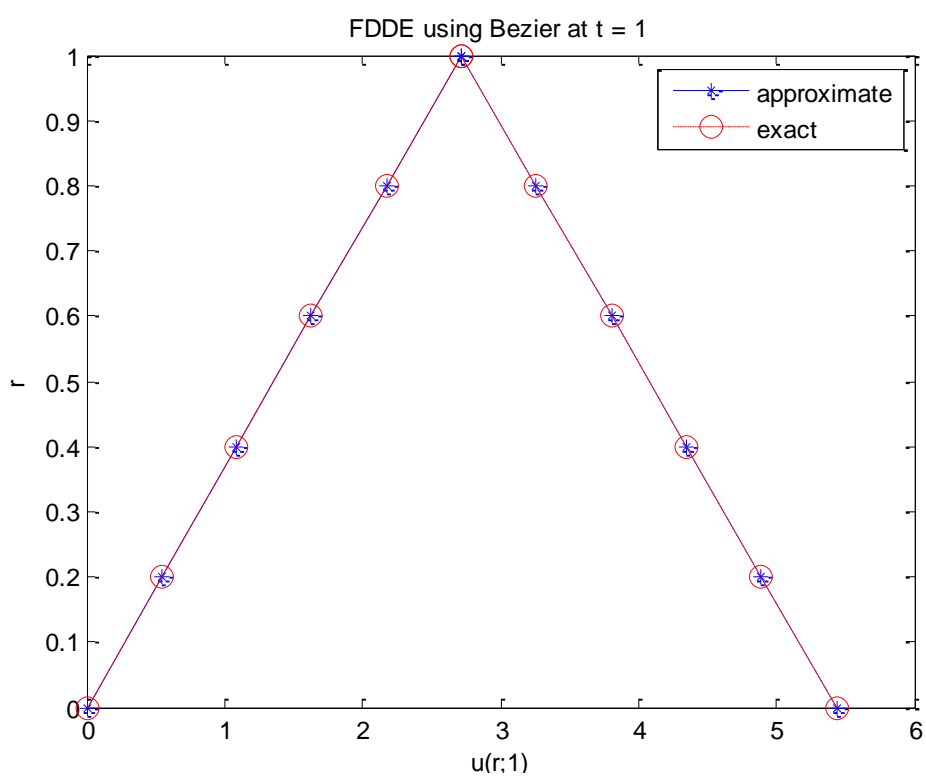

Figure 3. Approximate and exact solution of $u(t)$ at $\mathrm{t}=1$ (degree 8 bezier curves)

\section{CONCLUSION}

This work has successfully implemented and applied Bezier control points to overcome linear and fuzzy DDEs. A general method framework has been successfully developed and evaluated using fuzzy sets properties to obtain rough solutions for fuzzy DDEs. Details have been provided regarding the BCP convergence mechanism related to the approximate first-order fuzzy DDEs solution. Studies of first-order linear fizzy DDEs by BCP have shown that the system is capable and reliable studies are obtained that match the properties of the solution of the fizzy differential equation in the form of the triangle fuzzy numbers with varying degrees of precision.

\section{ACKNOWLEDGEMENTS}

The authors are very grateful to the Ministry of Higher Education of Malaysia for providing us with the Fundamental Research Grant Scheme (FRGS) S/O number 14188 to enable us to pursue this research.

\section{REFERENCES}

[1] G. Adomian and R. Rach, "Nonlinear Stochastic Differential Delay Equation,” Journal of Mathematical Analysis and Applications, vol. 91, no. 1, pp. 94-101, 1983.

[2] F. M. Asl and A. G. Ulsoy, "Analysis of a System of Linear Delay Differential Equations," Journal of Dynamic Systems, Measurement and Control, vol. 125, no. 2, pp. 215-223, 2003.

[3] K. A. G. Azevedo, "Existence and Uniqueness of Solution for Abstract Differential Equations with State-Dependent Time Impulses," Mediterranean Journal of Mathematics, vol. 16, no. 42. pp. 1-10, 2019.

[4] W. G. Aiello, et al., "Analysis of a model representing stage-structured population growth with state-dependent time delay," SIAM Journal on Applied Mathematics, vol. 52, no. 3, pp. 855-869, 1992.

[5] E. Ishiwata, "On the Attainable Order of Collocation Methods for the Neutral Functional-Differential Equations with Proportional Delays," Computing, vol. 64, pp. 207-222, 2000. 
[6] E. Ishiwata and Y. Muroya, "Rational Approximation Method for Delay Differential Equations with Proportional Delay," Applied Mathematics and Computation, vol. 187, no. 2, pp. 741-747, 2007.

[7] E. Ishiwata, et al., "A Super-Attainable Order in Collocation Methods for Differential Equations with Proportional Delay," Applied Mathematics and Computation, vol. 198, no. 1, pp. 227-236, 2008.

[8] P. Hu, et al., "Asymptotic Stability of Linear Multistep Methods for Nonlinear Neutral Delay Differential Equations," Applied Mathematics and Computation, vol. 211, no. 1, pp. 95-101, 2009.

[9] Z. Fan, et al., "Existence and Uniqueness of the Solutions and Convergence of Semi-Implicit Euler Methods for Stochastic Pantograph Equations," Journal of Mathematical Analysis and Applications, vol. 325, no. 2, pp. 1142-1159, 2007.

[10] W. Wang, et al., "Stability of One-Leg $\theta$ - Methods for Nonlinear Neutral Differential Equations with Proportional Delay," Applied Mathematics and Computation, vol. 213, no. 1, pp. 177-183, 2009.

[11] W. Wang, et al., "Stability of Continuous Runge-Kutta-Type Methods for Nonlinear Neutral Delay- Differential Equations," Applied Mathematical Modelling, vol. 33, no. 8, pp. 3319-3329, 2009.

[12] S. Z. Ahmad, et al., "Solving Oscillatory Delay Differential Equations Using Block Hybrid Methods," Journal of Mathematics, vol. 2018, pp. 285-301, 2018.

[13] A. K. Alomari, et al., "Solution of Delay Differential Equation by Means of Homotopy Analysis Method," Acta Applicandae Mathematicae, vol. 108, no. 2, pp. 395-412, 2009.

[14] S. Liao, "Series Solutions of Unsteady Boundary-Layer Flows Over a Stretching Flat Plate," Studies in Applied Mathematics, vol. 117, no. 3, pp. 239-263, 2006.

[15] D. J. Evans and K. R. Raslan, "The Adomian Decomposition Method for Solving Delay Differential Equation," International Journal of Computer Mathematics, vol. 82, no. 1, pp. 49-54, 2005.

[16] F. Shakeri and M. Dehghan, "Solution of Delay Diffrential Equation via a Homotopy Perturbation Method," Mathematical and Computer Modelling, vol. 48, no. 3-4, pp. 486-498, 2008.

[17] A. F. Jameel, et al., "A New Approximate Solution of the Fuzzy Delay Differential Equations," International Journal of Mathematical Modelling and Numerical Optimisation, vol. 9, no. 3, pp. 221-240, 2019.

[18] S. Narayanamoorthy and T. L. Yookesh, "Approximate method for solving the linear fuzzy delay differential equations," Discrete Dynamics in Nature and Society, vol. 2015, pp. 1-9, 2015.

[19] X. Guo, et al., "Fuzzy approximate solutions of second-order fuzzy linear boundary value problems," Journal of Boundary Value Problems, vol. 2013, pp. 1-17, 2013.

[20] L. A. Zadeh, “Toward a generalized theory of uncertainty," Information Sciences, vol. 172, no. 1-2, pp. 1-40, 2005.

[21] J. Biazar and B. Ghanbari, "The homotopy perturbation method for solving neutral functional-differential equations with proportional delays," Journal of King Saud University - Science, vol. 24, no. 1, pp. 33-37, 2012.

[22] X. Chen and L. Wang, "The Variational Iteration Method for Solving a Neutral Functional Differential Equation with Proportional Delays,” Computers and Mathematics with Applications, vol. 59, no. 8, pp. 2696-2702, 2010.

[23] T. Kushimoto and M. Hosaka, "Description of Intersection Curves by Differential Equation and their Tracing," Journal of the Japan Society for Precision Engineering, vol. 57, no. 8, pp. 1375-1380, 1991

[24] Wubshet I. and Getu K., "Magnetohydrodynamic flow of a nanofluid due to a non-linearly curved stretching surface with high order slip flow," Heat Transfer-Asian Research, vol. 48, no. 6, pp. 1-25, 2019.

[25] Z. C. Li and H. T. Huang, "Blending curves for landing problems by numerical differential equations II," Numerical methods, Computers \& Mathematics with Applications, vol. 39, no. 5-6, pp. 165-187, 2000. 